\title{
EUV-TEC proxy to describe ionospheric variability using satellite-borne solar EUV measurements
}

\author{
C. Unglaub ${ }^{1}$, Ch. Jacobi ${ }^{1}$, G. Schmidtke ${ }^{2}$, B. Nikutowski ${ }^{1,2}$, and R. Brunner ${ }^{2}$ \\ ${ }^{1}$ University of Leipzig, Institute for Meteorology, Stephanstr. 3, 04103 Leipzig, Germany \\ ${ }^{2}$ Fraunhofer IPM, Heidenhofstraße 8, 79110 Freiburg, Germany \\ Correspondence to: C. Unglaub (unglaub@uni-leipzig.de)
}

\begin{abstract}
An updated version of a proxy, termed EUV-TEC, describing the global total primary photoionisation is calculated from satellite-borne EUV measurements assuming a model atmosphere consisting of four major atmospheric constituents. Regional number densities of the background atmosphere are taken from the NRLMSISE-00 climatology. For calculation the Lambert-Beer law is used to describe the decrease of the radiation along their way through the atmosphere. The EUV-TEC proxy thus describes the ionospheric response to solar EUV radiation and its variability. EUV-TEC is compared against the global mean total electron content (TEC), a fundamental ionospheric parameter created from vertical TEC maps derived from GPS data. Strong correlation between these indices is found on different time scales. Results show that the EUV-TEC proxy represents the ionsopheric variability better than the conventional solar index F10.7 does, especially during high and moderate solar activity.
\end{abstract}

\section{Introduction}

The EUV (Extreme Ultraviolet) radiation is defined as the wavelength range between $10 \mathrm{~nm}$ and $121 \mathrm{~nm}$ (ISO, 2007). It is completely absorbed in the terrestrial atmosphere at altitudes above $50 \mathrm{~km}$. The absorption occurs mainly in the upper atmosphere, i.e. the thermosphere/ionosphere system, and therefore solar EUV radiation is the most important energy source at altitudes above $100 \mathrm{~km}$. It interacts with the atoms and molecules in this region through photodissociation and, at wavelengths up to $102 \mathrm{~nm}$, through photoionisation, thereby leading to the development of the planetary ionosphere. However, independent from the respective mechanism EUV radiation absorption finally causes heating of the thermosphere.
The total electron content (TEC) of the atmosphere is a fundamental ionospheric parameter defined as the electron density integrated along a path under consideration. To determine TEC, the ionospheric influence of radio wave propagation paths may be used, because the ionospheric effect on the propagation velocity depends on the radio wave frequency and the ionospheric electron density integrated along the radio wave propagation path. Because GPS-satellites emit two coherent frequencies the total electron content along the line of sight between the GPS satellite and a ground-based receiver can be deduced, and subsequently may be converted into vertical TEC. Thus measured TEC here is defined as the height integrated electron density between ground and the satellite orbit (Aggarwal, 2011).

Solar EUV radiation varies on different time-scales where the 11-yr Schwabe sunspot cycle causes the primary decadalscale irradiance variability and the Carrington rotation with an average period of 27 days causes the primary short-term variability. Consequences are strong changes of temperature, composition, density, electron density and ion content of the upper atmosphere. This can affect Low Earth Orbiting (LEO) satellites through variable atmospheric drag, and disturb communication and navigation signals (Woods, 2008).

The solar activity is often described by simple solar indices like the solar radio flux F10.7, which is defined as the solar radio emission at a wavelength of $10.7 \mathrm{~cm}$. However, the primary factor that controls TEC variations and the variability of thermospheric density and temperature is the solar EUV radiation (Emmert and Picone, 2010; Maruyama, 2010) and a nonlinear relationship between F10.7 and EUV fluxes has been found (Liu et al., 2011). Especially during the extended last solar minimum from 2007 to 2009, F10.7 is not an ideal proxy for solar EUV irradiance (Lühr and Xiong, 2010; Chen et al., 2011). Thus, there is a need for updated EUV indices to describe the ionospheric variability. 
In this paper we describe progress in constructing a new ionospheric proxy, EUV-TEC, which is intended to explain solar induced ionospheric variability, because the ionospheric electron content is primarily determined by the direct photoionisation induced by the incident solar EUV radiation (Lean et al., 2011). The proxy thus describes ionospheric, not solar variability, in response to the changing sun. The proxy may be used for space weather monitoring and ionospheric research. EUV-TEC is calculated from satellite-borne instruments measuring the EUV radiation considering the modified composition of the atmosphere which is caused by the EUV radiation. This proxy will be compared with F10.7 and the global mean TEC to demonstrate that the ionospheric variability is described better by EUV-TEC than conventional indices like F10.7.

\section{EUV-TEC calculation}

Solar EUV radiation is nearly completely absorbed in the upper atmosphere. It interacts with the atmospheric gas in this region and thus the EUV radiation will be attenuated. The decrease of the radiation along its propagation path is described by Lambert-Beer's law:

$\mathrm{d} I(\lambda, z)=I_{0}(\lambda) \cdot \sum_{i} \sigma_{i}(\lambda) \cdot n_{i}(z) \cdot \mathrm{d} s$,

where $\mathrm{d} I$ is the absorbed radiation along the radiation path $\mathrm{d} s$ through the atmosphere dependent on the local radiation flux $I_{0}$, the absorption cross section $\sigma_{i}$, both dependent on the wavelength $\lambda$ and the number densities $n_{i}$ of the respective gas.

EUV-spectra with a resolution of $1 \mathrm{~nm}$ are available from the Solar EUV Experiment (SEE) on board the TIMED satellite (Woods et al., 2000, 2005) since February 2002 to date. We use version 10 level 3 products available at LASP, University of Colorado, through http://lasp.colorado. edu/see/see_data.html. Additional EUV data are available from the SOLar Auto-Calibrating EUV/UV Spectrometers (SolACES) Experiment on the ISS since 2008 up to the present (Schmidtke et al., 2006a,b; Nikutowski et al., 2011) with a resolution of $1 \mathrm{~nm}$, too. SolACES has in-flight absolute calibration capability and therefore can be used for validation of other EUV data. However because of the orbit of the ISS a continous measurement is not possible so that only few EUV-spectra from SolACES are available.

For the calculation of EUV absorption and ionisation a spherical model atmosphere is assumed around a spherical model earth surface. The model atmosphere consists of the four major constituents $\mathrm{O}, \mathrm{N}, \mathrm{O}_{2}$ and $\mathrm{N}_{2}$. It reaches from the ground to an altitude of $1000 \mathrm{~km}$ with a resolution of $1 \mathrm{~km}$. The absorption and ionisation cross sections are taken from Metzger and Cook (1964) and Fennelly and Torr (1992) and were averaged to get a $1 \mathrm{~nm}$ resolution like the EUV-spectra have (Unglaub et al., 2011). For the calculation we assume that only photons contribute to the ionisation. Secondary ionisation processes are neglected.

An earlier version of EUV-TEC has been determined using globally averaged number densities (Unglaub et al., 2011). Now, regional thermospheric composition profiles from NRLMISE-00 (Picone et al., 2002) are used. To obtain the proxy, first a sphere with $6370 \mathrm{~km}$ radius is assumed surrounded by 1000 spherical shells with $1 \mathrm{~km}$ distance representing the atmospheric layers. Then, the points of intersection between the radiation paths of the incoming EUV-radiation and the spherical shells are determined. Subsequently these intersection points are converted into geographical coordinates considering the declination. Thus, the regional thermospheric densities can be calculated by the NRLMSISE-00 model for every particular atmospheric layer.

To calculate the primary ionisation rates, Eq. (1) is numerically integrated along each radiation path through the layers of the atmosphere. The path lengths $\mathrm{d} s$ through each particular layer is deduced from the intersection points between the radiation paths and the spherical shells. The primary ionisation is calculated for each layer along the radiation paths, integrated over one day and multiplied by the area where the radiation impacts. This results in the total ion production rate per day in the atmosphere. By dividing the production rate by the surface of the earth, the EUV-TEC proxy is obtained representing the global mean ion production per day and $\mathrm{m}^{2}$ in the atmosphere.

The regional thermospheric densities and the primary ionisation were calculated with a horizontal resolution of $220 \mathrm{~km}$ and a temporal resolution of $4 \mathrm{~h}$. This modified calculation and a refinement of the abort criterion causes a slightly larger daily ion production rate in the atmosphere than has been obtained by Unglaub et al. (2011).

\section{EUV-TEC proxy: Results}

To check how good EUV-TEC mirrors the ionospheric variability, the proxy has been compared against a global daily mean TEC created from gridded vertical TEC maps recorded with the IGS tracking network (Hernandez-Pajares et al., 2009). The datasets are available every $2 \mathrm{~h}$ for different longitudes and latitudes with a horizontal resolution of $2.5^{\circ}$. They were weighted with the cosine of their geographic latitude und thereafter a global diurnal mean was calculated. To compare the indices, EUV-TEC, TEC, and F10.7 data were each normalized by subtracting their mean value between July 2002 and June 2007 and dividing through their respective standard deviation. We chose this time period because EUV datasets from TIMED/SEE are available from February 2002 so a complete solar cycle is not available with a comparatively small data amount during solar maximum conditions that can be used for the normalization. This procedure also ensures that the peculiarities of the recent solar minimum are highlighted, since this time inteval is not 

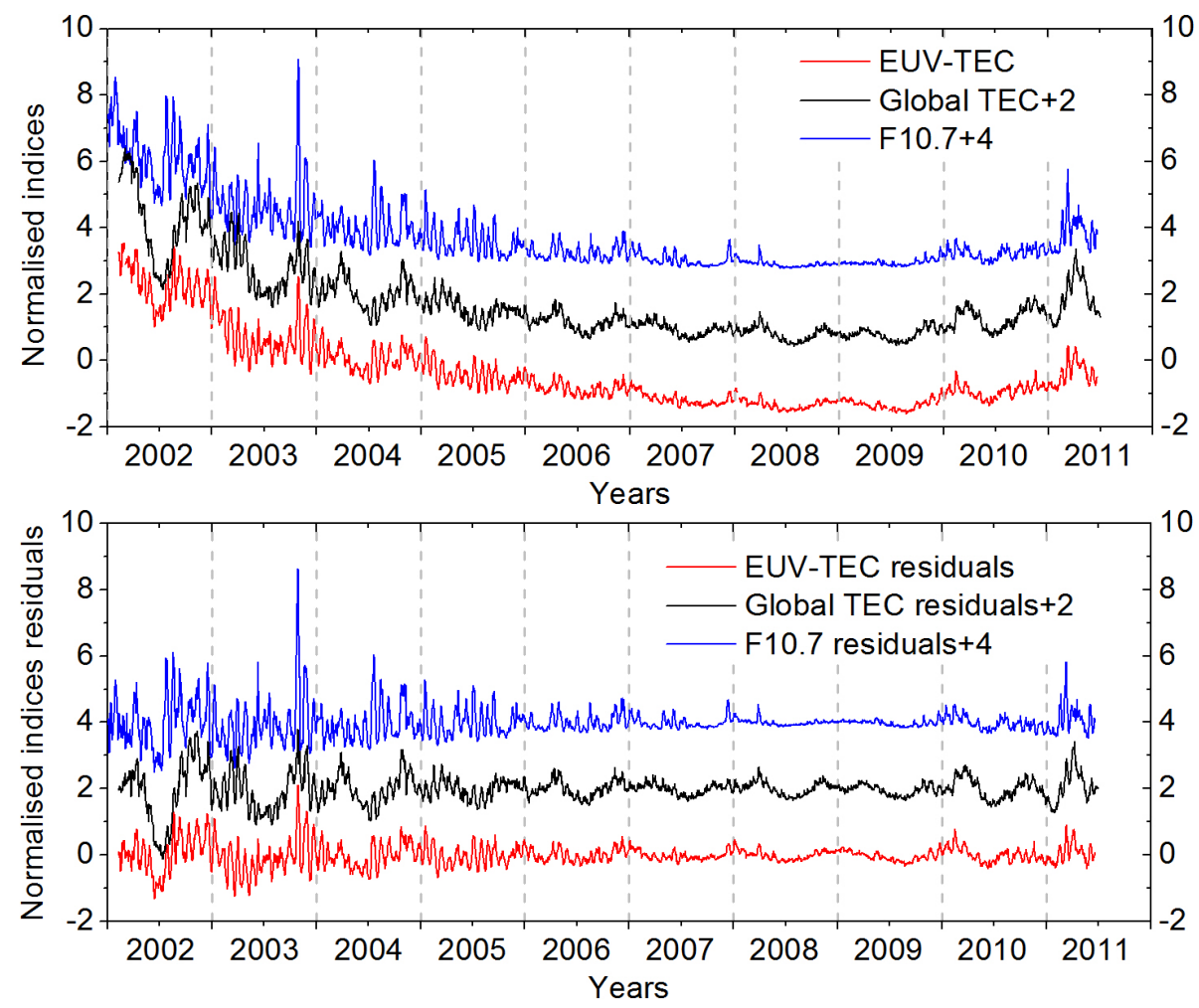

Fig. 1. February 2002 to June 2011 time series of the normalized EUV-TEC, global TEC and F10.7 (upper panel) and of the residuals after subtracting annual mean data (lower panel).

included in the data used for normalization. As a side effect, however, the average of the subsequently presented proxy data is not zero.

The time series of normalized EUV-TEC, normalized F10.7 and normalized global TEC are shown in Fig. 1 (upper panel). All data are uncorrected for earth orbit effect to study the solar influence on the atmosphere. Comparing the tree time series at low solar activity the seasonal pattern is well visible in both global TEC and EUV-TEC. In contrast during solar minimum F10.7 attains nearly consistent values showing a less marked seasonal pattern with a smaller amplitude than global TEC and EUV-TEC have. The seasonal pattern of EUV-TEC and F10.7 is mainly caused by the earth orbit effect where the earth is in perihelion at the beginning of January and in aphelion at the beginning of July. Thus, the indices attain the largest values at the turn of the year and the smallest values in the midyear. In addition to the earth orbit effect global TEC shows a half-year oscillation with two maxima in spring and autumn which can describe neither EUV-TEC nor F10.7, because it is dynamically induced.

Unglaub et al. (2011) have shown that a stronger correlation exists between the global mean TEC and the EUV-TEC proxy than between global TEC and F10.7 during 20022009. The updated EUV-TEC proxy shown in Fig. 1 is strongly correlated with global TEC, too, with a correlation coefficient of $r=0.95$, whereas the correlation coefficient between global TEC and F10.7 is $r=0.89$ for data from February 2002 to June 2011. Thus EUV-TEC describes the ionospheric variability, including long-term and short-term variability, better than F10.7 during 2002-2011. The strong correlations essentially result from the 11-yr solar cycle, because all indices attain smaller values with decreasing solar activity. To subtract this trend, the data were smoothed by adjacent averaging over 365 days and the smoothed values were subtracted from the normalized indices to get the residuals that describe only short-term variability and the seasonal cycle. These residuals are shown in Fig. 1 (lower panel).

In the left panel of Fig. 2 the normalized residuals of EUVTEC are shown vs. the normalized residuals of global TEC. A significant correlation between these indices with a correlation coefficient of $r=0.68$ is obtained. This correlation is sligthly stronger than one between the normalized residuals of the EUV-radidation in the wavelenght range from $5 \mathrm{~nm}$ to $102 \mathrm{~nm}$ and the normalized residuals of global TEC $(r=0.682$ vs. $r=0.679)$. The updated EUV-TEC proxy describes the ionospheric variability better than the earlier version of the EUV-TEC proxy ( $r=0.68$ vs. $r=0.67$ from 9 February 2002 to 31 December 2010) (Unglaub et al., 2011). The normalized residuals of F10.7 vs. the normalized residuals of global TEC are shown in the right panel of Fig. 2. There is a substantially weaker correlation than between the residuals of EUV-TEC and global TEC, with a correlation 

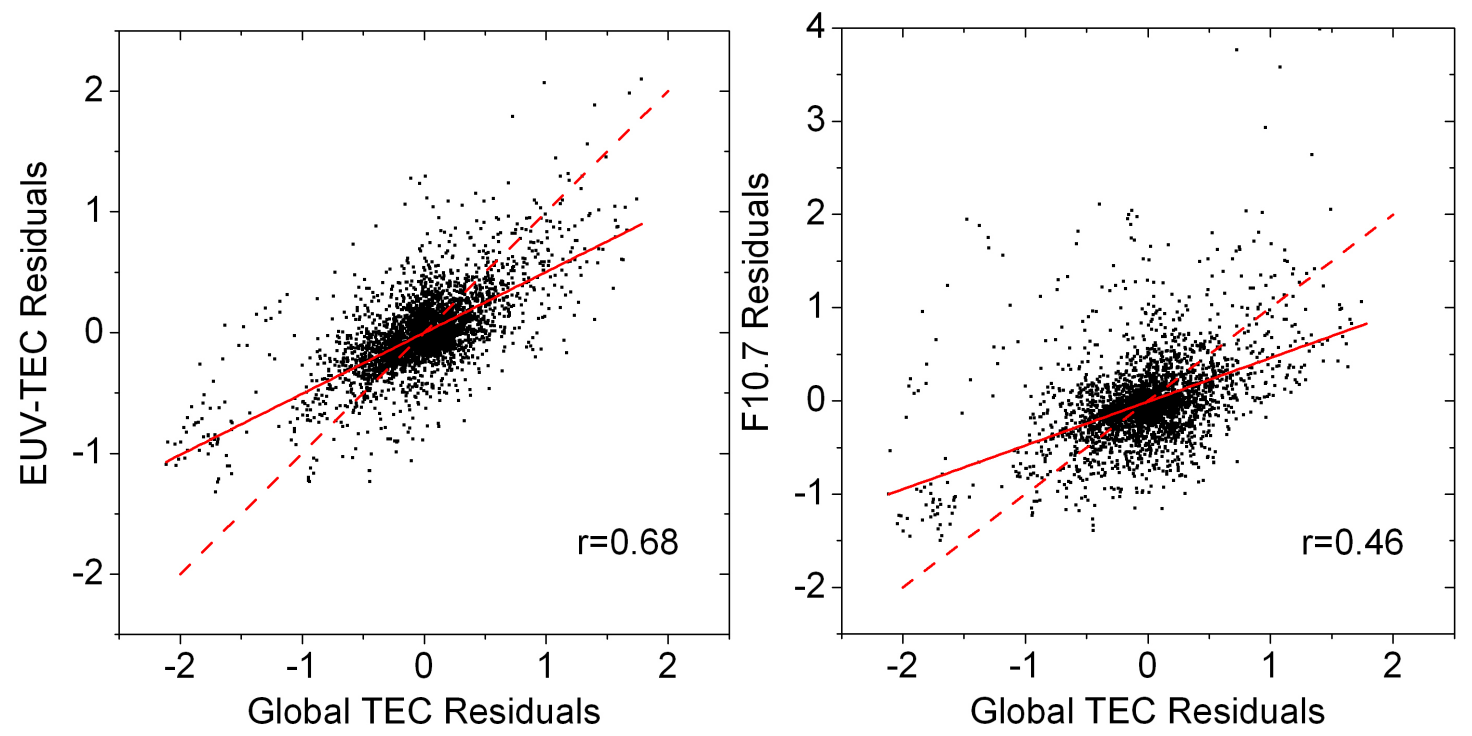

Fig. 2. Normalized EUV-TEC residuals (left panel) and normalized F10.7 residuals (right panel) vs. normalized global TEC residuals.

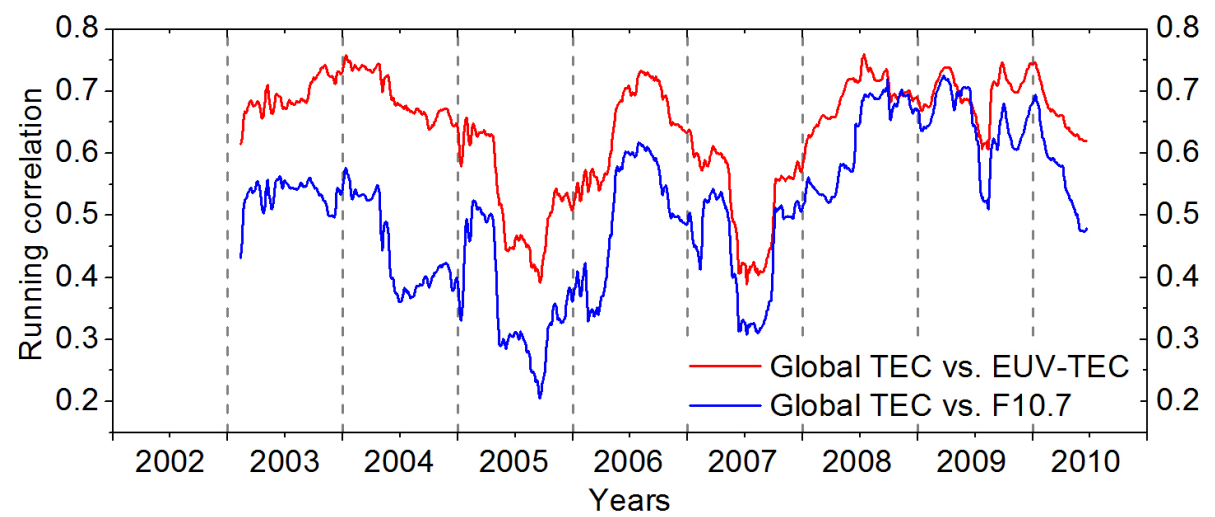

Fig. 3. Running correlations ( 365 days window) of the normalized global TEC residuals with the normalized EUV-TEC residuals (red line) and the normalized F10.7 residuals.

coefficient of $r=0.46$. Thus, during the time interval 2002 to 2011 the ionospheric short-term TEC variability and its seasonal pattern are clearly better described by EUV-TEC than by F10.7. Because of the dynamical influence the seasonal pattern of global TEC has larger amplitudes than the seasonal pattern of EUV-TEC and F10.7 has. This can be seen as well in Fig. 2.

Figure 1 also reveals that on the one hand during solar minimum both the curves of EUV-TEC and global TEC are smooth, although they show partly different seasonal cycles, such that the semiannual global mean TEC signature, which is a dynamical feature, is not represented in EUV-TEC. On the other hand, EUV-TEC and global TEC short-term variability connected with the Carrington rotation, partly differ during high and moderate solar activity. To investigate possible changes of correlation in the course of the seasonal cycle, in Fig. 3 the running correlations, each based on 365 days of data, between the normalized global TEC residuals and the normalized EUV-TEC residuals (red curve) or the normalized F10.7 residuals (blue curve), respectively, are shown. A continuously stronger correlation between the normalized global TEC residuals and the normalized EUV-TEC residuals than between the normalized global TEC residuals and the normalized F10.7 residuals exists during high and moderate solar activity. The correlation between global TEC and F10.7 partly becomes as small as $r=0.2$ while the correlation between global TEC and EUV-TEC is stronger, with $r>0.35$. However, for solar minimum conditions the correlations are similar. As a consequence, EUV-TEC describes ionospheric short-term variability better than F10.7 during high and moderate solar activity.

For solar minimum conditions the seasonal pattern is the dominant source of variability, because the sun is very quiet resulting in weak fluctuations. The similar correlations 
indicate that on an annual time scale F10.7 represents the seasonal pattern of global TEC as well as EUV-TEC does, merely with a different amplitude.

\section{Conclusions}

From satellite borne solar EUV measurements a new version of the EUV-TEC proxy, representing global mean photoionisation rates, have been calculated. The regional number densities of the background atmosphere are taken from the NRLMSISE-00 climatology. The EUV-TEC proxy describes the influence of solar variability on the ionosphere, and therefore can be considered as an ionospheric proxy, which may be used for the analysis of space weather effects on the upper atmosphere. It was compared with a global mean TEC created from vertical TEC maps. EUV-TEC shows a strong correlation with global TEC. Stronger correlations between EUV-TEC and global TEC than between the conventional solar index F10.7 and global TEC are found on different time scales. During 2002-2011 EUV-TEC describes the ionospheric variability, including both short-term and longterm variability, slightly better than F10.7 does. On an annual time scale EUV-TEC represents the short-term variability of the ionosphere distinctly better than F10.7 does, especially during high and moderate solar activity. On the whole, the EUV-TEC proxy performs better than F10.7 to describe ionospheric variability.

Acknowledgements. TIMED-SEE data has been provided by LASP, University of Colorado, through http://lasp.colorado.edu/see/see data.html. TEC data has been provided by NASA through ftp: //cddis.gsfc.nasa.gov/gps/products/ionex/. F10.7 indices have been provided by NGDC through ftp://ftp.ngdc.noaa.gov/STP/SOLAR_ DATA/.

Topical Editor Matthias Förster thanks Norbert Jakowski and an anonymous reviewer for their help in evaluating this paper.

\section{References}

Aggarwal, M.: TEC variability near northern EIA crest and comparison with IRI model, Adv. Space Res., 48, 1221-1231, 2011.

Chen, Y., Liu, L., and Wan, W.: Does the F10.7 index correctly describe solar EUV flux during the deep solar minimum of 2007-2009?, J. Geophys. Res., 116, A04304, doi:10.1029/2010JA016301, 2011.

Emmert, J. T. and Picone, J. M.: Climatology of globally averaged thermospheric mass density, J. Geophys. Res., 115, A09326, doi:10.1029/2010JA015298, 2010.

Fennelly, J. A. and Torr, D. G.: Photoionization and photoabsorption cross sections of $\mathrm{O}, \mathrm{N}_{2}, \mathrm{O}_{2}$ and $\mathrm{N}$ for aeronomic calculations, Atom. Data Nucl. Data Tables, 51, 321-363, 1992.
Hernandez-Pajares, M., Juan, J. M., Sanz, J., Orus, R., Garcia-Rigo, A., Feltens, J., Komjathy, A., Schaer, S. C., and Krankowski, A.: The IGS VTEC maps: a reliable source of ionospheric information since 1998, J. Geod., 83, 263-275, 2009.

ISO: ISO 21348:2007(E), Space environment (natural and artificial) - Process for determining solar irradiances, ISO, p. 12, 2007.

Lean, J. L., Meier, R. R., Picone, J. M., and Emmert J. T.: Ionospheric total electron content: Global and hemispheric climatology, J. Geophys. Res., 116, A10318, doi:10.1029/2011JA016567, 2011.

Liu, L., Chen, Y., Le, H., Kurkin, V. I., Polekh, N. M., and Lee, C.C.: The ionosphere under extremely prolonged low solar activity, J. Geophys. Res., 116, A04320, doi:10.1029/2010JA016296, 2011.

Lühr, H. and Xiong, C.: IRI-2007 model overestimates electron density during the 23/24 solar minimum, Geophys. Res. Lett., 37, L23101, doi:10.1029/2010GL045430, 2010.

Maruyama, T.: Solar proxies pertaining to empirical ionospheric total electron content models, J. Geophys. Res., 115, A04306, doi:10.1029/2009JA014890, 2010.

Metzger, P. H. and Cook, G. R.: A reinvestigation of the absorption cross sections of molecular oxygen in the 1050-1800 A region, J. Quant. Spectrosc. Radiat. Transfer, 4, 107-116, 1964.

Nikutowski, B., Brunner, R., Erhardt, Ch., Knecht, St., and Schmidtke, G.: Distinct EUV minimum of the solar irradiance (16-40 nm) observed by SolACES spectrometers onboard the International Space Station (ISS) in August/September 2009, Adv. Space Res., 48, 899-903, 2011.

Picone, J. M., Hedin, A. E., and Drob, D. P.: NRLMSISE00 empirical model of the atmosphere: statistical comparisons and scientic issues, J. Geophys. Res., 107, 1468, doi:10.1029/2002JA009430, 2002.

Schmidtke, G., Fröhlich, C., and Thuillier, G.: ISS-SOLAR: Total (TSI) and spectral (SSI) irradiance measurements, Adv. Space Res., 37, 255-264, 2006a.

Schmidtke, G., Brunner, R., Eberhard, D., Halford, B., Klocke, U., Knothe, M., Konz, W., Riedel, W.-J., and Wolf, H.: SOL-ACES: Auto-calibrating EUV/UV spectrometers for measurements onboard the International Space Station, Adv. Space Res., 37, 273 282, 2006b.

Unglaub, C., Jacobi, Ch., Schmidtke, G., Nikutowski, B., and Brunner, R.: EUV-TEC proxy to describe ionospheric variability using satellite-borne solar EUV measurements: first results, Adv. Space Res., 47, 1578-1584, 2011.

Woods, T. N.: Recent advances in observations and modeling of the solar ultraviolet and X-ray spectral irradiance, Adv. Space Res., 42, 895-902, 2008.

Woods, T. N., Bailey, S., Eparvier, F., Lawrence, G., Lean, J., McClintock, B., Roble, R., Rottmann, G. J., Solomon, S. C., Tobiska, W. K., and White, O. R.: TIMED Solar EUV Experiment, Phys. Chem. Earth (C), 25, 393-396, 2000.

Woods, T. N., Eparvier, F., Bailey, S., Chamberlin, P., Lean, J., Rottmann, G. J., Solomon, S. C., Tobiska, W. K., and Woodraska, D. L.: Solar EUV Experiment (SEE): Mission overview and first results, J. Geophys. Res., 110, A01312, doi:10.1029/2004JA010765, 2005. 